\title{
Efeito de Erros de Pedigree na Seleção ${ }^{1}$ \\ Paulo Luiz Souza Carneiro ${ }^{2}$, Ricardo Frederico Euclydes ${ }^{3}$, Martinho de Almeida e Silva ${ }^{4,5}$, Paulo Sávio Lopes ${ }^{3,5}$, Robledo de Almeida Torres ${ }^{3}$, Antônio Policarpo Souza Carneiro ${ }^{2}$, Rodolpho de Almeida Torres Filho6
}

\begin{abstract}
RESUMO - Dados simulados com cinco níveis de erros de pedigree $(0,5,10,15$ e $20 \%)$ e três herdabilidades $(0,10 ; 0,30$; e 0,60$)$ foram usados para verificar o efeito de erros de pedigree na seleção por 10 gerações consecutivas. Os maiores valores fenotípicos e de eficiência sobre a seleção individual, em geral, foram obtidos com o BLUP sem erro, seguidos daqueles com 5, 10, 15 e 20\% de erro. A superioridade média em ganho genético do BLUP por geração, nos vários níveis de erros, sobre a seleção individual, decresceu com o aumento da herdabilidade da caraterística. Na medida em que se elevaram os níveis de erros, o BLUP apresentou maior redução no ganho genético para as características com menores herdabilidades.
\end{abstract}

Palavras-chave: BLUP, ganho genético, seleção individual, simulação

\section{Effect of Pedigree Errors on the Selection}

\begin{abstract}
Data simulated with five levels of pedigree errors $(0,5,10,15$ and 20\%) and three heritabilities (.10, .30 and .60) were used to verify the effect of pedigree errors on the selection during 10 generations. The largest phenotypic values and efficiency on the individual selection, in overall, were obtained by BLUP without errors, followed by those with 5, 10, 15 and 20\% of error. The average superiority in genetic gain of the BLUP per generation, in the various error levels, on the individual selection, decreased with the increase of the heritability of the trait. As the error level increased, the BLUP presented bigger reduction on the genetic gain for the traits with smaller heritabilities.
\end{abstract}

Key Words: BLUP, genetic gain, individual selection, simulation

\section{Introdução}

Muitos métodos de avaliação genética têm sido desenvolvidos com o objetivo de selecionar os melhores animais para a reprodução. Quando as informações de pedigree contêm erros e os procedimentos utilizados levam em consideração informações de parentes, a aplicação destes métodos pode levar a interpretações errôneas.

VAN VLECK (1970a e 1970b) mostrou que, durante o processo de seleção, a identificação incorreta de progenitores bovinos pode provocar estimativas viesadas de herdabilidades e de progresso genético. Segundo RON et al. (1996), a taxa de erro de paternidade, usando grupos sangüíneos ou proteínas do leite, em bovinos, variou entre 4 e $23 \%$ na Alemanha; 5 e $15 \%$ na Dinamarca; 8 e $30 \%$ na antiga União Soviética; $1,3 \%$ no Reino Unido; e em uma população de gado Irlandês ficou em torno de $20 \%$ para machos e 8\% para fêmeas. No Brasil, ROSA et al. (1997) efetuaram teste de paternidade, usando marcadores genéticos RFLP Kapa-caseína ( $\mathrm{KpCn}$ ), b-lactoglobulina (bLG) e hormônio de crescimento $(\mathrm{GH})$ e os microssatélites IGF I, INRA-006, CSFM-50 e BM1224. Foram avaliados 63 animais da raça Nelore, não aparentados em primeiro grau, provenientes de 23 rebanhos, constatando-se que de 13 famílias (touro/vaca/ progênie) duas (15\%) acusaram erro de identificação.

Em suínos, LONG et al. (1990) citaram que o remanejo de leitões entre as leitegadas, prática muito comum para se obterem leitegadas mais uniformes, resulta potencialmente em maiores erros de pedigree que em bovinos. Ressalta-se que esses autores trabalharam com dados reais e utilizaram apenas uma geração de seleção para comparar a seleção individual, o índice de seleção e o BLUP, na presença de diferentes níveis de erros de pedigree, para características com herdabilidades de 0,$13 ; 0,18$; e 0,53.

Outro importante aspecto do uso de dados com erros de identificação é sua influência no teste de

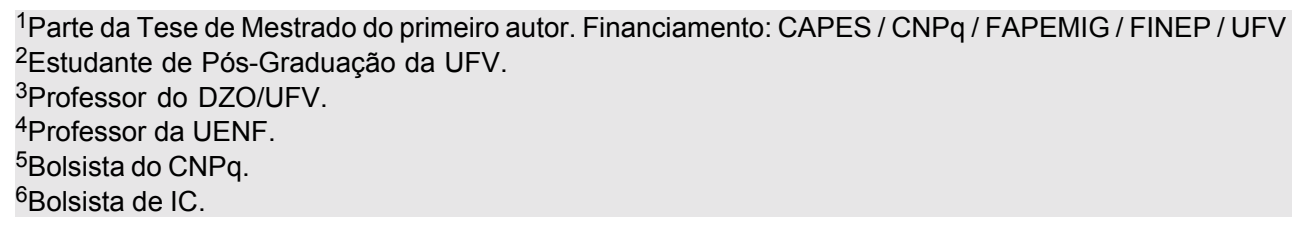


progênie. VAN VLECK (1970a e 1970b) cita que para os melhoristas torna-se importante saber como os erros de pedigree influenciam a precisão do teste de progênie e diminuem a resposta à seleção. Este autor mostra que erros de identificação de touros e vacas é problema grave, que reduz o progresso genético possível pela seleção, por meio de redução da correlação entre o verdadeiro valor genético e o predito. Kaup (1983) e Tiessem (1983), citados por GELDERMANN (1986), ressaltam a grande importância econômica da redução dos erros de identificação, chamando a atenção para a influência da mãode-obra utilizada no processo de inseminação artificial sobre o número de identificações erradas. GELDERMANN (1986) salienta que, pelo fato de os touros utilizados nos programas de inseminação artificial serem utilizados em número muito grande de vacas, erros de identificação mesmo em pequena percentagem podem ter grande efeito na taxa de ganho genético. Nesse caso, seria necessário o incremento do tamanho do grupo de progênies para se obter o mesmo ganho. Nos atuais programas de melhoramento, mais especificamente de suínos e aves, os erros de identificação ocorrem basicamente no campo, quando da coleta dos dados e durante o processo de digitação dos mesmos.

A metodologia de modelos mistos, para a obtenção da melhor predição linear não-viesada (BLUP), proposta por HENDERSON (1963, 1973 e 1974), apresentada por MARTINS et al. (1997), é um procedimento sujeito à redução na sua eficiência, quando os dados utilizados contêm erros de pedigree, pois utiliza informação de família por meio matriz de parentesco em modelo animal.

O objetivo deste trabalho foi verificar a eficiência do BLUP na presença de diferentes níveis de erros de pedigree durante várias gerações de seleção e sua comparação com a seleção individual, utilizando populações simuladas.

\section{Material e Métodos}

Os dados utilizados neste trabalho foram simulados com o programa GENESYS (EUCLYDES, 1996), o qual é constituído de nove programas, escrito para o compilador FORTRAN, e permite a simulação de genomas de certa complexidade para estudos de métodos de seleção, testes de pressuposições, avaliações de novas metodologias de seleção, entre outros. Maiores detalhes sobre o programa GENESYS são apresentados por EUCLYDES e GUIMARÃES (1997).
Foi simulado um genoma constituído de uma única característica quantitativa governada por 500 loci. O genoma simulado para o presente estudo apresentou os seguintes parâmetros: quinze pares de cromossomos de tamanhos aleatórios; três mil centimorgans de comprimento; os efeitos aditivos dos locos quantitativos foram simulados, segundo a distribuição normal; os locos quantitativos foram dialélicos e não possuíam desvios de dominância nem interações entre si; não possuíam cromossoma sexual e as freqüências gênicas iniciais eram iguais em ambos os sexos; a distribuição matemática usada para a simulação das freqüências iniciais dos locos quantitativos nas populações foi a uniforme, com média de 0,50 ; os efeitos fixos foram sexo, EF1 e EF2, sendo os dois últimos possuidores de 5 e 4 níveis ou classes, respectivamente; e os efeitos de ambiente não controláveis foram simulados conforme a distribuição normal.

A partir deste genoma foram simuladas três populações bases, nas quais a característica quantitativa possuía herdabilidade inicial de 0,10;0,30; e 0,60, respectivamente. Foram simuladas populações bases constituídas de 800 animais (400 machos e 400 fêmeas).

Para construção das populações iniciais, partiuse das três populações bases, correspondentes às herdabilidades estudadas, e escolheram-se, de forma aleatória, 20 machos e 200 fêmeas, que foram utilizados como progenitores das gerações seguintes. Para cada fêmea foram simulados oito descendentes, resultando em populações de 1.600 animais. Estas populações foram utilizadas para incorporação dos erros de pedigree e avaliação dos métodos de seleção.

Para obter os níveis de $0,5,10,15$ e $20 \%$ de erros de pedigree sobre os registros, implementou-se no GENESYS um programa capaz de simular erros completos no pedigree (pai e mãe). De cada uma das três populações iniciais simuladas para as herdabilidades 0,$10 ; 0,30$; e 0,60 , produziram-se sete cópias. Destas, uma foi usada como população controle, uma submetida à seleção individual e cinco submetidas à seleção baseada no BLUP, por dez gerações consecutivas.

Para as populações submetidas ao BLUP adotaram-se os seguintes procedimentos: em uma população, usada como referência, não foram introduzidos erros de pedigree $(0 \%)$; para simular as populações com $5,10,15$ e $20 \%$ de erros, número equivalente às percentagens de erros dos filhos foram redistribuídos, aleatoriamente, para outros pais, ou seja, para a população com $5 \%$ de erros de pedigree, do total de 1.600 animais das populações, 80 tiveram seus regis- 
tros de paternidade alterados. Para os níveis 10, 15 e $20 \%$, o número de animais com registros de paternidade alterados foram 160, 240 e 320, respectivamente.

A cada geração foram selecionados 20 machos e 200 fêmeas que foram acasalados ao acaso, sendo que cada macho foi acasalado com 10 fêmeas e de cada acasalamento foram produzidos oito descendentes. Este processo foi conduzido durante 10 gerações consecutivas e por 100 ciclos, ou repetições, com o objetivo de reduzir os efeitos das flutuações gênicas, sendo os vários níveis de erros de pedigree incorporados às populações a cada geração.

Os métodos de escolha dos animais para serem pais da geração seguinte e utilizados com o objetivo de avaliar os efeitos dos erros de pedigree sobre a estimativa de ganhos genéticos foram:

- Escolha ao acaso, em que os reprodutores foram amostrados, de forma totalmente aleatória, independente de qualquer informação fenotípica ou genotípica;

- Seleção individual (IND), em que os reprodutores foram selecionados segundo seus valores fenotípicos. Para seleção baseada apenas no fenótipo do indivíduo, usou-se um índice $\left(I_{\mathrm{i}}\right)$ que correspondia ao valor fenotípico padronizado $\left(X_{j i}^{*}\right)$ desta característica, da seguinte forma:

$$
I_{i}=X_{j i}^{*} \quad \text { e } \quad X_{j i}^{*}=\frac{\left(X_{j i}-\bar{X}_{j}\right)}{\sigma_{x j}}
$$

em que $X_{j i}$ representa o valor fenotípico do indivíduo $i$ na população com herdabilidade $j ; \bar{X}_{j}$, a média fenotípica para a população com herdabilidade $j$; e $\sigma_{x j}$, o desvio-padrão fenotípico para população com herdabilidade $j$. Para cada macho e cada fêmea foi calculado um valor de $I_{\mathrm{i}}$. De posse dos valores de $\mathrm{I}_{\mathrm{i}}$ de cada animal, procedeu-se à seleção, ordenandose os 20 melhores machos e as 200 melhores fêmeas, que foram utilizados como os pais da geração seguinte;

- BLUP - melhor predição linear não-viesada, em que os candidatos à reprodução foram selecionados segundo informações do seu fenótipo e de seus parentes. Para escolha dos melhores animais, baseada no BLUP, foi utilizada a metodologia de modelos mistos e os dados foram analisados utilizando-se o seguinte modelo estatístico:

$$
y_{i j k l}=\mu+A_{i}+B_{j}+S_{k}+g_{l(i j k)}+e_{l(i j k)}
$$

em que

$\mathrm{y}_{\mathrm{ijk}, \mathrm{k}}=$ observação do animal $l$, do sexo $k$ referentes aos efeitos fixos EF1 $i$ e EF2 $j ; \mu=$ constante associada à todas observações; $A_{i}=$ efeito fixo do $\mathrm{EF} 1, \operatorname{com} i=1,2,3,4,5 ; B_{j}=$ efeitos fixo do EF2, com $\mathrm{j}=1,2,3,4 ; S_{k}=$ efeito fixo do sexo $k, \operatorname{com} k=1,2$; $g_{l(j k)}=$ efeito aleatório do indivíduo $l$, no EF $1 i, \mathrm{EF} 2 j \mathrm{e}$ sexo $k$; e $e_{l(j k)}=$ erro aleatório associado a cada observação.

$\mathrm{Na}$ escolha dos animais para reprodução (20 machos e 200 fêmeas), selecionaram-se os animais que obtiveram maior $\hat{g}$, que representa o valor genético predito do indivíduo, em cada uma das populações avaliadas.

Nas populações com 0, 5, 10, 15 e 20\% de erros, as eficiências do BLUP em relação à seleção individual (método não influenciado por erros de pedigree) e ao BLUP com $0 \%$ erro de pedigree, foram avaliadas por meio das seguintes expressões:

$$
E_{B I t}=\frac{\Delta G_{B t}}{\Delta G_{l t}} \text { e } E_{B B t}=\frac{\Delta G_{B t}}{\Delta G_{B(0) t}}
$$

em que $E_{B t}$ e $E_{B B t}$ representama eficiência do BLUP, em relação à seleção individual e em relação ao BLUP com $0 \%$ de erro na geração t; $\Delta G_{B t}$ é o ganho genético obtido pelo BLUP na geração t para os diferentes níveis de erros; $\Delta G_{l t}$ é o ganho genético obtido pela seleção individual na geração t; e $\Delta G_{B(0) t}$ é ganho genético obtido pelo BLUP com $0 \%$ de erro na geração t.

\section{Resultados e Discussão}

As populações que tiveram os pais escolhidos ao acaso, populações-controle (PCON), apresentaram pequena oscilação no valor fenotípico durante as dez gerações de seleção a que foram submetidas (Tabela 1). Este comportamento, característico de flutuações gênicas, foi semelhante ao observado por EUCLYDES (1996), que comparou métodos de seleção para diferentes densidades de marcadores moleculares.

Os maiores valores fenotípicos e os maiores valores de eficiência sobre a seleção individual, em geral, foram obtidos com o BLUP sem erro, seguidos daqueles com 5, 10, 15 e 20\% de erro (Tabelas 1 e 2). Entretanto, para populações com herdabilidade inicial 0,60 , pequena diferença foi observada entre os valores fenotípicos nas 10 gerações simuladas, havendo pequeno decréscimo na eficiência para os dados com erros. Isto se deve ao fato de o BLUP, por intermédio do modelo animal e das equações de modelos mistos, fornecer menos peso às informações de parentes, quando a característica trabalhada apresenta alta herdabilidade, sendo, portanto, neste caso, pouco influenciado por erros de pedigree. Por outro lado, as características com herdabilidade 0,10 e 0,30 foram bastante influenciadas, mostrando a grande impor- 
272 Rev. bras. zootec.

Tabela 1 - Valores fenotípicos observados ao longo das gerações de seleção

Table 1 - Observed phenotypic values along the generations of selection

Método de seleção Geração

Selection method

Generation

\begin{tabular}{|c|c|c|c|c|c|c|c|c|c|c|}
\hline & 1 & 2 & 3 & 4 & 5 & 6 & 7 & 8 & 9 & 10 \\
\hline & \multicolumn{10}{|c|}{ Herdabilidade $($ Heritability $)=0,10$} \\
\hline$\overline{B L U P}(0 \%)^{1}$ & $-0,5$ & 6,2 & 15,2 & 24,1 & 32,7 & 40,7 & 48,9 & 56,5 & 64,2 & 71,7 \\
\hline $\operatorname{BLUP}(5 \%)^{1}$ & $-0,5$ & 6,2 & 14,9 & 23,4 & 31,9 & 40,0 & 47,8 & 55,5 & 62,7 & 69,5 \\
\hline $\operatorname{BLUP}(10 \%)^{1}$ & $-0,5$ & 5,7 & 14,3 & 22,3 & 30,4 & 38,5 & 46,2 & 53,7 & 60,1 & 66,8 \\
\hline $\operatorname{BLUP}(15 \%)^{1}$ & $-0,5$ & 5,5 & 13,6 & 21,9 & 30,0 & 37,6 & 45,2 & 52,6 & 59,5 & 66,1 \\
\hline $\operatorname{BLUP}(20 \%)^{1}$ & $-0,5$ & 5,3 & 12,7 & 20,2 & 27,8 & 35,2 & 42,5 & 49,3 & 56,2 & 63,0 \\
\hline $\mathrm{IND}^{2}$ & $-7,0$ & $-1,1$ & 4,7 & 10,5 & 16,0 & 21,6 & 27,3 & 33,0 & 38,5 & 43,9 \\
\hline \multirow[t]{2}{*}{$\mathrm{PCON}^{3}$} & $-11,2$ & $-11,4$ & $-11,4$ & $-11,1$ & $-11,0$ & $-10,8$ & $-10,7$ & $-10,7$ & $-10,6$ & $-10,6$ \\
\hline & \multicolumn{10}{|c|}{ Herdabilidade $($ Heritability $)=0,30$} \\
\hline $\operatorname{BLUP}(0 \%)$ & 1,9 & 12,0 & 23,6 & 35,5 & 47,0 & 57,9 & 68,3 & 77,8 & 86,9 & 95,5 \\
\hline BLUP(5\%) & 1,9 & 12,0 & 23,8 & 35,0 & 45,5 & 56,2 & 66,1 & 75,6 & 84,9 & 93,8 \\
\hline BLUP(10\%) & 1,9 & 11,8 & 23,4 & 34,7 & 45,2 & 55,6 & 65,3 & 75,2 & 84,5 & 93,2 \\
\hline BLUP $(15 \%)$ & 1,9 & 11,7 & 22,8 & 33,5 & 44,1 & 54,4 & 64,2 & 74,0 & 83,3 & 92,2 \\
\hline BLUP(20\%) & 1,9 & 11,5 & 22,5 & 33,4 & 44,0 & 54,2 & 64,0 & 73,4 & 82,2 & 90,9 \\
\hline IND & $-0,3$ & 9,6 & 19,5 & 28,9 & 38,3 & 47,5 & 56,4 & 65,2 & 74,0 & 82,3 \\
\hline \multirow[t]{2}{*}{ PCON } & $-11,2$ & $-11,4$ & $-11,4$ & $-11,1$ & $-11,0$ & $-10,8$ & $-10,7$ & $-10,7$ & $-10,6$ & $-10,6$ \\
\hline & \multicolumn{10}{|c|}{ Herdabilidade $($ Heritability $)=0,60$} \\
\hline $\operatorname{BLUP}(0 \%)$ & $-4,6$ & 8,9 & 22,9 & 36,7 & 50,11 & 63,14 & 75,5 & 87,20 & 98,2 & 109,3 \\
\hline BLUP(5\%) & $-4,6$ & 8,9 & 22,8 & 36,6 & 50,0 & 62,9 & 75,3 & 87,1 & 98,3 & 108,9 \\
\hline BLUP(10\%) & $-4,6$ & 8,7 & 22,6 & 36,3 & 49,7 & 62,7 & 75,0 & 87,0 & 98,3 & 109,0 \\
\hline BLUP(15\%) & $-4,61$ & 8,7 & 22,5 & 36,1 & 49,3 & 62,1 & 74,4 & 85,9 & 97,2 & 108,1 \\
\hline BLUP(20\%) & $-4,6$ & 8,8 & 22,7 & 36,4 & 49,7 & 62,7 & 75,3 & 87,2 & 98,6 & 109,5 \\
\hline IND & $-7,8$ & 6,2 & 19,6 & 32,7 & 45,0 & 58,2 & 71,7 & 83,7 & 94,6 & 105,1 \\
\hline PCON & $-11,2$ & $-11,4$ & $-11,4$ & $-11,1$ & $-11,0$ & $-10,8$ & $-10,7$ & $-10,7$ & $-10,6$ & $-10,6$ \\
\hline
\end{tabular}

1 BLUP = Melhor Predição Linear Não-Viesada com diferentes níveis de erro de pedigree.

2 IND = Seleção individual.

3 PCON = População sem seleção.

1 BLUP = Best Linear Unbiased Prediction with differents levels of pedigree errors.

2 IND = Individual selection.

3 PCON = Unselected population.

Tabela 2 - Eficiência do BLUP em relação à seleção individual

Table 2 - Efficiency of BLUP in relation to individual selection

Método de seleção

Selection method

Geração

\begin{tabular}{|c|c|c|c|c|c|c|c|c|c|c|}
\hline \multirow[t]{2}{*}{ Selection method } & \multicolumn{10}{|c|}{ Generation } \\
\hline & 1 & 2 & 3 & 4 & 5 & 6 & 7 & 8 & 9 & 10 \\
\hline & \multicolumn{10}{|c|}{ Herdabilidade $($ Heritability $)=0,10$} \\
\hline $\operatorname{BLUP}(0 \%)^{1}$ & 2,35 & 1,68 & 1,63 & 1,60 & 1,60 & 1,57 & 1,55 & 1,52 & 1,51 & 1,49 \\
\hline $\operatorname{BLUP}(5 \%)^{1}$ & 2,35 & 1,68 & 1,61 & 1,57 & 1,57 & 1,55 & 1,52 & 1,50 & 1,48 & 1,45 \\
\hline $\operatorname{BLUP}(10 \%)^{1}$ & 2,35 & 1,63 & 1,58 & 1,52 & 1,51 & 1,50 & 1,48 & 1,46 & 1,42 & 1,41 \\
\hline $\operatorname{BLUP}(15 \%)^{1}$ & 2,35 & 1,61 & 1,53 & 1,51 & 1,50 & 1,47 & 1,45 & 1,43 & 1,41 & 1,39 \\
\hline \multirow[t]{2}{*}{$\operatorname{BLUP}(20 \%)^{1}$} & 2,35 & 1,59 & 1,48 & 1,43 & 1,42 & 1,40 & 1,38 & 1,36 & 1,35 & 1,34 \\
\hline & \multicolumn{10}{|c|}{ Herdabilidade $($ Heritability $)=0,30$} \\
\hline $\operatorname{BLUP}(0 \%)$ & 1,20 & 1,11 & 1,13 & 1,16 & 1,17 & 1,17 & 1,17 & 1,16 & 1,15 & 1,14 \\
\hline BLUP(5\%) & 1,20 & 1,11 & 1,14 & 1,15 & 1,14 & 1,14 & 1,14 & 1,13 & 1,12 & 1,12 \\
\hline BLUP $(10 \%)$ & 1,20 & 1,10 & 1,12 & 1,14 & 1,14 & 1,13 & 1,13 & 1,13 & 1,12 & 1,11 \\
\hline BLUP $(15 \%)$ & 1,20 & 1,10 & 1,10 & 1,11 & 1,11 & 1,11 & 1,11 & 1,11 & 1,10 & 1,10 \\
\hline \multirow[t]{2}{*}{ BLUP $(20 \%)$} & 1,20 & 1,09 & 1,09 & 1,11 & 1,11 & 1,11 & 1,11 & 1,10 & 1,09 & 1,09 \\
\hline & \multicolumn{10}{|c|}{ Herdabilidade $($ Heritability $)=0,60$} \\
\hline $\operatorname{BLUP}(0 \%)$ & 1,69 & 1,14 & 1,10 & 1,08 & 1,08 & 1,06 & 1,04 & 1,03 & 1,03 & 1,03 \\
\hline $\operatorname{BLUP}(5 \%)$ & 1,69 & 1,14 & 1,10 & 1,08 & 1,08 & 1,06 & 1,04 & 1,03 & 1,03 & 1,03 \\
\hline $\operatorname{BLUP}(10 \%)$ & 1,69 & 1,13 & 1,09 & 1,07 & 1,08 & 1,06 & 1,03 & 1,03 & 1,03 & 1,03 \\
\hline $\operatorname{BLUP}(15 \%)$ & 1,69 & 1,13 & 1,09 & 1,07 & 1,07 & 1,05 & 1,03 & 1,02 & 1,02 & 1,02 \\
\hline $\operatorname{BLUP}(20 \%)$ & 1,69 & 1,13 & 1,09 & 1,08 & 1,08 & 1,06 & 1,04 & 1,03 & 1,03 & 1,03 \\
\hline
\end{tabular}

${ }^{1}$ BLUP $=$ Melhor Predição Linear Não-Viesada com diferentes níveis de erro de pedigree.

${ }^{1}$ BLUP = Best Linear Unbiased Prediction with different levels of pedigree errors. 
CARNEIRO et al.

tância dada pela metodologia de modelos mistos às informações de parentes para características com herdabilidades menores.

Pode-se verificar pela Tabela 2 que, mesmo na presença de erros de pedigree, o BLUP foi mais eficiente que a seleção individual durante todas as gerações estudadas. Quando se comparou a eficiência do BLUP na presença dos vários níveis de erros com o BLUP sem erros, observaram-se decréscimos nos ganhos genéticos ao longo das gerações (Tabela 3 ). A superioridade média em ganho genético por geração nos vários níveis de erros do BLUP sobre a seleção individual decresceu com o aumento da herdabilidade da caraterística (Tabela 4). À medida que se aumentaram os níveis de erros, o BLUP apresentou maior redução no ganho genético para herdabilidades menores (Tabela 5).

Os resultados apresentados mostraram que o BLUP é influenciado por erros de pedigree, principalmente, para características de baixa herdabilidade, em que o BLUP valoriza mais as informações de parentes em detrimento às informações do próprio indivíduo. Estes resultados foram similares aos observados por LONG et al. (1990), que trabalharam com uma única geração de seleção, comparando o BLUP com outros métodos de seleção na presença de erros de pedigree. Esses autores observaram vantagens nas expectativas de ganhos genéticos do
BLUP sem erros de pedigree sobre a seleção individual de 30,8;22,0; e 7,2\%, para características com herdabilidades de 0,$13 ; 0,18$; e 0,53 , respectivamente. Em relação ao BLUP sem erros de pedigree, o BLUP com $20 \%$ de erro apresentou reduções de 12,4; 9,3; e $3,8 \%$ na expectativa de ganhos genéticos, para as herdabilidades 0,$13 ; 0,18$; e 0,53 , respectivamente.

Os resultados obtidos neste estudo, para dados sem erros de pedigree, foram similares aos observados por BELONSKY e KENNEDY (1988), que em rebanho de suínos simulado, com 100 matrizes por um período de 10 anos, comparando a seleção individual com o BLUP para características com herdabilidades 0,$10 ; 0,30$; e 0,60 , encontraram vantagens no uso do BLUP sobre a seleção individual de, respectivamente, 55, 25 e 10\%. KUHLERS e KENNEDY (1992), ao usarem o BLUP e a seleção pelo fenótipo para selecionarem os animais de reposição, verificaram que a seleção com o BLUP acarretou 72, 36, e $12 \%$ a mais de ganho genético para herdabilidades 0,$10 ; 0,30$; e 0,60 , respectivamente, que a seleção pelo fenótipo do indivíduo, após 10 gerações de seleção. Esta superioridade do BLUP, na escolha dos melhores animais para reprodução, para dados sem erros de pedigree, foi demonstrada em vários outros trabalhos (KEELE et al., 1988; SORENSEN, 1988; VERRIER et al., 1993; e JEYRUABAN et al., 1995).

Tabela 3 - Eficiência do BLUP com erros de pedigree em relação ao BLUP com $0 \%$ de erro

Table 3 - Efficiency of BLUP, with pedigree errors in relation to BLUP with $0 \%$ of error

Método de seleção $\quad$ Geração

Selection method Generation

\begin{tabular}{|c|c|c|c|c|c|c|c|c|c|c|}
\hline & 1 & 2 & 3 & 4 & 5 & 6 & 7 & 8 & 9 & 10 \\
\hline & \multicolumn{10}{|c|}{ Herdabilidade $($ Heritability $)=0,10$} \\
\hline $\operatorname{BLUP}(0 \%)^{1}$ & 1,0 & 1,0 & 1,0 & 1,0 & 1,0 & 1,0 & 1,0 & 1,0 & 1,0 & 1,0 \\
\hline $\operatorname{BLUP}(5 \%)^{1}$ & 1,0 & 1,0 & 0,98 & 0,98 & 0,98 & 0,98 & 0,98 & 0,98 & 0,98 & 0,97 \\
\hline $\operatorname{BLUP}(10 \%)^{1}$ & 1,0 & 0,97 & 0,96 & 0,94 & 0,94 & 0,95 & 0,95 & 0,95 & 0,94 & 0,94 \\
\hline $\operatorname{BLUP}(15 \%)^{1}$ & 1,0 & 0,96 & 0,94 & 0,93 & 0,93 & 0,94 & 0,93 & 0,94 & 0,93 & 0,93 \\
\hline \multirow[t]{2}{*}{$\operatorname{BLUP}(20 \%)^{1}$} & 1,0 & 0,90 & 0,89 & 0,90 & 0,89 & 0,88 & 0,89 & 0,89 & 0,89 & 0,89 \\
\hline & \multicolumn{10}{|c|}{ Herdabilidade $($ Heritability) $=0,30$} \\
\hline BLUP $(0 \%)$ & 1,0 & 1,0 & 1,0 & 1,0 & 1,0 & 1,0 & 1,0 & 1,0 & 1,0 & 1,0 \\
\hline BLUP $(5 \%)$ & 1,0 & 1,0 & 1,0 & 0,98 & 0,97 & 0,97 & 0,97 & 0,97 & 0,97 & 0,98 \\
\hline BLUP $(10 \%)$ & 1,0 & 0,99 & 0,99 & 0,98 & 0,96 & 0,96 & 0,96 & 0,97 & 0,97 & 0,97 \\
\hline $\operatorname{BLUP}(15 \%)$ & 1,0 & 0,98 & 0,97 & 0,95 & 0,94 & 0,94 & 0,94 & 0,95 & 0,96 & 0,96 \\
\hline \multirow[t]{2}{*}{$\operatorname{BLUP}(20 \%)$} & 1,0 & 0,97 & 0,96 & 0,95 & 0,94 & 0,94 & 0,94 & 0,95 & 0,95 & 0,95 \\
\hline & \multicolumn{10}{|c|}{ Herdabilidade $($ Heritability $)=0,60$} \\
\hline BLUP $(0 \%)$ & 1,0 & 1,0 & 1,0 & 1,0 & 1,0 & 1,0 & 1,0 & 1,0 & 1,0 & 1,0 \\
\hline BLUP $(5 \%)$ & 1,0 & 1,0 & 0,99 & 0,99 & 0,99 & 0,99 & 0,99 & 0,99 & 0,99 & 0,99 \\
\hline BLUP $(10 \%)$ & 1,0 & 0,99 & 0,99 & 0,99 & 0,99 & 0,99 & 0,99 & 0,99 & 0,99 & 0,99 \\
\hline BLUP(15\%) & 1,0 & 0,99 & 0,98 & 0,98 & 0,98 & 0,98 & 0,98 & 0,98 & 0,98 & 0,98 \\
\hline $\operatorname{BLUP}(20 \%)$ & 1,0 & 0,99 & 0,99 & 0,99 & 0,99 & 0,99 & 0,99 & 0,99 & 1,0 & 1,0 \\
\hline
\end{tabular}

${ }^{1}$ BLUP = Melhor Predição Linear Não-Viesada com diferentes níveis de erro de pedigree.

${ }^{1}$ BLUP $=$ Best Linear Unbiased Prediction with different levels of pedigree errors. 
274 Rev. bras. zootec.

Tabela 4 - Superioridade média em ganho genético por geração obtida pelo BLUP, com 0, 5, 10, 15 e $20 \%$ de erro de pedigree, sobre a seleção individual

Table 4 - Mean superiority in genetic gain by generation obtained by BLUP with 0, 5, 10, 15 and $20 \%$ of pedigree errors on the individual selection

\begin{tabular}{lccc}
\hline $\begin{array}{l}\text { Método de seleção } \\
\text { Selection method }\end{array}$ & \multicolumn{3}{c}{$\begin{array}{c}\text { Herdabilidade } \\
\text { Heritability }\end{array}$} \\
\cline { 2 - 4 } & 0,10 & 0,30 & 0,60 \\
\hline BLUP(0\%) & 65,4 & 16,1 & 13,4 \\
BLUP(5\%) & 63,2 & 14,4 & 13,2 \\
BLUP $(10 \%)^{1}$ & 59,1 & 13,6 & 12,9 \\
BLUP(15\%) & 57,1 & 12,1 & 12,3 \\
BLUP(20\%) & 51,5 & 11,4 & 13,1 \\
\hline
\end{tabular}

${ }^{1}$ BLUP $=$ Melhor Predição Linear Não-Viesada com diferentes níveis de erro de pedigree.

${ }^{1}$ BLUP = Best Linear Unbiased Prediction with different levels of pedigree errors.

Tabela 5 - Redução média em ganho genético por geração obtido pelo BLUP, com $0,5,10,15$ e $20 \%$ de erro de pedigree, em relação ao BLUP com $0 \%$ de erro

Table 5 - $\quad$ Mean reduction in the genetic gain by generation (\%) obtained y BLUP with 0, 5, 10, 15 and $20 \%$ of pedigree errors, in relation to BLUP with $0 \%$ of error

\begin{tabular}{llcl}
\hline $\begin{array}{l}\text { Método de seleção } \\
\text { Selection method }\end{array}$ & \multicolumn{3}{c}{$\begin{array}{c}\text { Herdabilidade } \\
\text { Heritability }\end{array}$} \\
\cline { 2 - 4 } & 0,10 & 0,30 & 0,60 \\
\hline BLUP $(0 \%)^{1}$ & 0,0 & 0,0 & 0,0 \\
BLUP $(5 \%)^{1}$ & 1,5 & 1,5 & 0,2 \\
BLUP $(10 \%)^{1}$ & 4,1 & 2,1 & 0,5 \\
BLUP $(15 \%)^{1}$ & 5,3 & 3,5 & 1,1 \\
BLUP $(20 \%)^{1}$ & 8,9 & 4,0 & 0,3 \\
${ }^{1}$ BLUP = Melhor Predição Linear Não-Viesada com diferentes \\
níveis de erro de pedigree. \\
${ }^{1}$ BLUP = Best Linear Unbiased Prediction with different levels of pedigree \\
errors.
\end{tabular}

\section{Conclusões}

Com base nestes resultados, conclui-se que a metodologia BLUP é bastante influenciada por erros de pedigree, principalmente, para características de baixa herdabilidade, em que as informações de parentes são mais importantes. Entretanto, a seleção de animais, por intermédio desta metodologia, mesmo na presença de erros de pedigree, proporcionou maiores ganhos genéticos que a seleção individual.

Com relação à redução em ganho genético provocado pela inclusão de erros de pedigree, conclui-se que, para as características com herdabilidades menores, esta redução foi bastante acentuada, mostrando a necessidade de maior rigidez no controle das identificações dos animais. Já para características com herdabilidades altas $(0,60)$, a inclusão de erros de pedigree não provocou redução nos ganhos genéticos.

\section{Referências Bibliográficas}

BELONSKY, G. M., KENNEDY, B. W. 1988. Selection on individual phenotype and best linear unbiased predictor of breeding value in a closed swine herd. J. Anim. Sci., 66:1124-1131.

EUCLYDES, R.F. Uso do sistema para simulação Genesys na avaliação de métodos de seleção clássicos e associados a marcadores moleculares. Viçosa, MG: Universidade Federal de Viçosa, 1996. 149 p. Tese (Doutorado em Genética e Melhoramento) - Universidade Federal de Viçosa, 1996.

EUCLYDES, R. F., GUIMARÃES, S.E.F. 1997. Associação dos métodos tradicionais de seleção à seleção assistida por marcadores moleculares. R. Bras. Reprod. Anim., 21(3):89-96.

GELDERMANN, H., PIEPER, U., WEBER, W. E. 1986. Effect of misidentification on the estimation of breeding value and heritability in cattle. J. Anim. Sci., 63:1759-1768.

HENDERSON, C.R. 1963. Selection index and expected genetic advance. In: NATIONAL ACADEMY OF SCIENCE/ NATIONAL RESEARCH COUNCIL - NAS/NRC. Statistical genetics and plant breeding. - Washington, DC, p. 141-163.

HENDERSON, C.R. Sire evaluation and genetic trends. In: ANIMAL BREEDING GENETIC SYMPOSIUM IN HONOR OF Dr. J. L. LUSH, 1973, Blacksburg. Proceedings... ASAS/ADSA, p.10-41, 1973.

HENDERSON, C.R. 1974. General flexibility of linear model techniques for sire evaluation. J. Dairy Sci., 57:963-972.

JEYARUBAN, M. G., GIBSON, J. P., GOWE, R. S. 1995. Comparison of index selection and best linear unbiased prediction for simulated layer poultry data. Poult. Sci., 74:1566-1576.

KEELE, J. W., JOHNSON, R. K., YOUNG, L. D. et al. 1988. Comparison of methods of predicting breeding values of swine. J. Anim. Sci., 66:3040-3048.

KUHLERS, D. L., KENNEDY, B. W. 1992. Effect of culling on selection response using phenotypic selection or best linear unbiased prediction of breeding values in small, closed herds of swine. J. Anim. Sci., 70:2338-2348.

LONG, T.E., JOHNSON, R.K., KEELE, J.W. 1990. Effects of erros in pedigree on three methods of estimating breeding value for litter size, backfat and average daily gain in swine. J. Anim. Sci., 68:4069-78.

MARTINS, E.N., LOPES, P.S., SILVA, M.A. et al. 1997. Uso de modelos mistos na avaliação genética animal. Cadernos Didáticos, Viçosa: UFV, n.18, 121p.

RON, M., BLANC, Y., BAND, M. et al. 1996. Misidentification rate in the Israeli dairy cattle population and its implications for genetic improvement. J. Dairy Sci., 79:676-681.

ROSA, A. J. M., PACKER, I. U., REGITANO, L. C. A. et al. Caracterização da raça Nelore e teste de paternidade por marcadores moleculares. In: REUNIÃO ANUAL DA REVISTA SOCIEDADE BRASILEIRA DE ZOOTECNIA, 34, 1997, Juiz de Fora. Anais... Juiz de Fora: EMBRAPA, 3:6264, 1997.

SORENSEN, D.A. 1988. Effect of selection index versus mixed model methods of prediction of breeding value on response to selection in a simulated pig population. Livest. Prod. Sci., 20:135-148.

VAN VLECK, L.D. 1970b. Misidentification in estimating the paternal sib correlation. J. Dairy Sci., 53:1469-1474, 1970a.

VAN VLECK, L.D. Misidentification and sire evaluation. $J$. Dairy Sci., 53:1697-1702.

VERRIER, E., COLLEAU, J. J., FOULlEY, J. L. 1993. Longterm effects of seletion based on the animal model BLUP in a finite population. Theor. Appl. Genet., 87:446-454.

Recebido em: 18/06/98

Aceito em: 26/10/98 